Proposition of: paper

\title{
Functionalised silicon oxide nanoparticles for fingermark detection
}

\author{
Sébastien Moret ${ }^{1,2}$, Andy Bécue ${ }^{2}$, Christophe Champod $^{2}$
}

1 Centre for Forensic Science, University of Technology Sydney, Broadway, 2007, Australia

2École des Sciences Criminelles, Building Batochime, University of Lausanne, CH-1015

Lausanne, Switzerland

* Corresponding Author

Dr. Sébastien Moret

Centre for Forensic Science

University of Technology Sydney

PO Box 123, Broadway, 2007

Australia

Phone: 0061295142758

E-mail: sebastien.moret@uts.edu.au

Authors contact details:

Sébastien Moret: $\quad$ sebastien.moret@uts.edu.au

Andy Bécue: $\quad$ andy.becue@unil.ch

Christophe Champod: christophe.champod@unil.ch 


\section{Abstract:}

2 Over the past decade, the use of nanotechnology for fingermark detection has been attractinga

3 lot of attention. A substantial number of nanoparticle types has thus been studied and applied 4 with varying success.However, despite all efforts, few publications presentclear supporting 5 evidence of their superiority over standard and commonly used techniques. This paper 6 focuses on a rarely studied type of nanoparticles that regroups all desired properties for 7 effective fingermark detection: silicon oxide. These nanoparticles offer optical and surface 8 properties that can be tuned to provide optimal detection. This study explores their potential 9 as a new method for fingermark detection.

10 Detection conditions, outer functionalisations and optical properties were optimised and a 11 first evaluation of the technique is presented. Dye-doped silicon oxide nanoparticles were 12 assessed against a one-step luminescent cyanoacrylate. Both techniques were compared on 13 natural fingermarks from three donors collected on four different non-porous substrates. On 14 average, the two techniques performed similarly but silicon oxide detected marks with a 15 better homogeneity and was less affected by donor inter-variability. The technique remains to 16 be further optimised and yet silicon oxide nanoparticles already show great promises for 17 effective fingermark detection.

19 Keywords: Nanotechnology, luminescence, sensitivity, selectivity, cyanoacrylate, non20 porous substrates. 


\section{Introduction}

23 Nanoparticles applied for fingermark detection are known to offer several advantages over 24 traditional methods[1]. First, their small size may lead to detect marks with a high resolution 25 without risking over-development that can typically arise with conventional techniques such as powder dusting or cyanoacrylate fuming. Second, somenanoparticles possess interesting luminescent properties spread over a broad area of the electromagnetic spectrum ranging from UV to infrared. Conventional luminescence properties, as well as up-conversion can be used to mitigate substrate interferences. Infrared luminescence is also of great interest for problematic surfaces since few materials are optically active in this range of wavelengths[2].

31 Finally, and more importantly, the surface of nanoparticles can be precisely tuned to offer a large panel of potential interaction with fingermarks. By grafting molecules or functional groups onto their surfaces, it becomes possible to specifically target various components of the fingermark residue, leading to an increased selectivity. Fingermarks left bysmokers could thus be distinguished from the marks left by non-smokers[3].Altogether, these propertiesshow great promises forfingermark detection, and can lead toan overall increase ofboth sensitivity and selectivity.

Over the past decade, these advantages attracted a lotof attention andasubstantial number of nanoparticle types have been studied and appliedwith varying success. They can be sorted into three categories, i.e. metal, metal oxide and semi-conductors.Gold and silver nanoparticles have been successfully used in techniques such as multi- or single-metal deposition (gold)[4, 5] or physical developer (silver)[6]. Among metal oxides, we can cite titanium dioxide $\left(\mathrm{TiO}_{2}\right)$ [7], aluminium oxide $\left(\mathrm{Al}_{2} \mathrm{O}_{3}\right)$ [8, 9] or zinc oxide ( $\left.\mathrm{ZnO}\right)$ [10]. Metal oxides are generally applied as dried powders, sometimes functionalised with aliphatic chains to increase and favour hydrophobic interactions with greasy components of the fingermark residue.Quantum dots are the most studied class of semiconductors. They attracted a lot of attention mostly for their uncommon optical properties $[11,12]$. This paper does not intend to describethese nanoparticles in extensive details; thoroughreviews are available elsewhere [13, 14].

However, from a critical viewpoint, none of the nanoparticles studied until nowand their subsequent applicationsentirely fulfil the criteria described above. Some have a size over 100 $\mathrm{nm}$, and cannot really be classify as nanoparticles according to international [15] and national

53 [16] organisations. Even if not everyone agrees on the $100 \mathrm{~nm}$ limit, sub-micron particles 54 would be a more appropriate denomination for particles of a size above $200 \mathrm{~nm}$.A similar 55 commentcan be madefornano-sized powderobtained after solvent evaporation. It is not 
56 obvious that these nanoparticles are not agglomeratedinto larger clusters once dried. In that 57 case, properties arising from the nanometric-size such as luminescence may be retained, but 58 detection with a high resolution related with nanometric materialwill belost. Moreover, among nanoparticles used up-to-now few possess intrinsic luminescent properties. Most metallic andmetal-oxide nanoparticles present weak if no luminescence at all, restricting their applications to light-coloured substrates only. Some attempts have been made to confer 62 luminescent properties to those nanoparticles types. For example, zinc oxide layer can be 63 added aroundgold nanoparticles to get luminescent fingermark[17]. However, this application 64 remains limited to non-porous substrates and implies a tedious protocol.Another option is to 65 coat nanoparticles with a luminescent dye $[8,9,18]$.Among intrinsic luminescent nanoparticles, quantum dots are the most commonly cited. These semi-conductor nanoparticles are luminescent under UV excitation with a narrow emission peak. Their emission colour is directly related to the size of the particles. This particular property is due to quantum confinement effect $[19,20]$ and has driven a lot of endeavour towards their use as a new tool for fingermark detection[21-25, 26 ]. However, despite all efforts, few publications presentclear supporting evidence of their superiority over standard and commonly used techniques.Cost, toxicity and tedious synthetic procedures set aside, the main problem with quantum dots is the difficulty to properly functionalise their surface without altering the structural properties and compromising the luminescence properties. Very few occurrences of successfully and specifically functionalised particles for fingermark detection can be found. Some quantum dots have been functionalised with carboxyl or amine groups[27], but despite the presented results, the real effect of the surface modification on the detection properties remains unclear. This emphasisesthe fact that even if particles are nanosized and possess interesting optical properties, their surface still has to offer extensive functionalisation properties in order to be successfully used for fingermark detection.

81 Hence, despite all the nanoparticle types applied for fingermark detection so far, none of them reallybenefit at the same time from the three advantages presented above (i.e. small size, optical properties and surface modifications). One kind of nanoparticles - yet underrepresented in the literature - could regroupall the properties in one singleentity: the silicon oxide nanoparticles $\left(\mathrm{SiO}_{2}\right)$. This paper focuses on them and explores how they can offer potential as a method for fingermark detection. Silicon oxide nanoparticles consist of a 87 porous matrix of siloxane bonds, with an external layer of silanol groups that can further reactthrough hydrolysis and condensation with variousalkoxysilanes, leading to a

89 functionalised layer covalently bound to the main matrix. As a 
90 consequence, $\mathrm{SiO}_{2}$ nanoparticles could present an almost unlimited range of functional groups.

91 Dye molecules can be entrapped within thesiloxane matrix during the synthesis, providing a wide range ofoptical properties. Various synthetic procedures exist, but two of them are most commonly considered: the Stöber's synthesis and the reversed micro-emulsion. The Stöber's synthesis[28]leads to bulk production, but the size and surface controls are limited. On the other end, reversed micro-emulsion[29] enables to accurately control size, optical properties and functionalisation all at the same time, but with a lower yield.

$97 \mathrm{SiO}_{2}$ nanoparticles have been studied and successfully applied for fingermark detection on 98 very rare occasions and with focus mostly put towards optical properties. Theaker et al. 99 investigated the use of hydrophobic micro- and nanoparticles containing sub-particlesas well 100 as rhodamine 6G among other dyes [30]. Applied as a dried powder or in suspension,these 101 particles were shown to detect fingermarks on several non-porous substrates such as glass or stainless steel. Finely grounded xerogelwas synthesised using either a europium complex [31]

103 or rhodamine B [32]. Each of these dyeswas usedas powder dusting to detect marks. More 104 recently, $700 \mathrm{~nm}$ amphiphilic $\mathrm{SiO}_{2}$ particles were appliedon fingermarks on glass 105 microscopic slides but without any particular dyes embedded in their matrix[33].

106 In a recent study $\mathrm{SiO}_{2}$ nanoparticles functionalised with various chemical groups and 107 dispersed in aqueous solutionswere used to specifically study the interaction occurring between fingermark residues and nanoparticles [34]. It was demonstrated that chemical interaction between carboxyl and amine groupscould be promotedfollowing three different procedures:by lowering the $\mathrm{pH}$ of the solution, by adding sodium chloride $(\mathrm{NaCl})$ to decrease

111 zeta potential intensity of the nanoparticles or by adding a diimide compound that activates 112 the amide linkage formation.

114 The study was focused on understanding the mechanism involved during the detection rather 115 than on the quality of the resulting detected mark. We think that gaining a better 116 understanding of the mechanism is a prerequisite to any work towards optimising the 117 method.This present study aims at exploring the possibilities offered by $\mathrm{SiO}_{2}$ nanoparticles in 118 terms of a new tool for fingermark detection, by optimising and comparing various detection 119 conditions, outer functionalisations and luminescent dyes.More work still needs to be 120 undertaken in order to provide a new fully operational technique, but theresults obtained 121 during this study showed that $\mathrm{SiO}_{2}$ nanoparticles are very promising and that research effort 122 should be further pursued. 


\section{2. Materials and methods}

$125 \quad 2.1$ Synthesis and functionalisation of $\mathrm{SiO}_{2}$ nanoparticles

$126 \mathrm{SiO}_{2}$ nanoparticles were synthetised via reversed micro-emulsion where droplets of water 127 containing dye act as microreactors, allowing nanoparticles growth. Even if this method is 128 not the most cost-effective and has a low yield, it allowed synthetising nanoparticles with an 129 acute size control, introducing a dye in the matrix and functionalising nanoparticles surface, 130 all in a single synthetic process. Once nanoparticles were synthesised and functionalised, they 131 were precipitated out of the emulsion, washed and dissolved in water.

132 The same synthetic procedure described in Moret et al. was followed without any 133 modification [34].Tetraethyl orthosilicate (TEOS), Triton X-100 (TX-100), cyclohexane, n134 hexanol, ammonium hydroxide (25 wt \%), rhodamine $6 \mathrm{G}$, rhodamine $\mathrm{B}$, tris $\left(2,2^{\prime}\right.$ 135 bipyridyl)dichlororuthenium (II) hexahydrate (RuBpy)and sodium chloride ( $\mathrm{NaCl}$ ) were 136 purchased from Sigma-Aldrich. Carboxyethylsilanetriol sodium salt (CES) and 3137 (triethoxysilyl)-propylsuccinic anhydride (TES-PSA) were purchased from Abcr GmbH \& 138 Co. All chemicals were used as received without further purification and were stable for 139 several months under storage conditions recommended by manufacturer. Briefly, the 140 synthetic procedure consisted in mixing $3.54 \mathrm{~mL}$ of $\mathrm{TX}-100,15 \mathrm{~mL}$ of cyclohexane and 3.6 $141 \mathrm{~mL}$ n-hexanol in a round bottom flask. $960 \mu \mathrm{L}$ of a water-based dye solution were then added. Three luminescent dyes (rhodamine 6G, rhodamine B and RuBpy) were tested in order to compare optical properties. The dye concentration of the solution added during the syntheses was $100 \mathrm{mM}$ for both rhodamine $6 \mathrm{G}$ and rhodamine $\mathrm{B}$, but for stability reasons it was lowered to $16.6 \mathrm{mM}$ for RuBpy, as recommended by Bagwe et al.[35].After stabilisation of the micro-emulsion, $200 \mu \mathrm{L}$ of tetraethyl orthosilicate and $120 \mu \mathrm{L}$ of ammonium hydroxide were successively added to initiate nanoparticles formation. The mixture was then

148 kept under stirring for $24 \mathrm{~h}$.

149 For the functionalisation step, two different silane coupling agents were used for comparison purposed,CES and TES-PSA(Table 1),both containing carboxyl groups.This step was conducted directly in the micro-emulsion mixture by adding $50 \mu \mathrm{L}$ of tetraethyl orthosilicate along with $170 \mu \mathrm{mol}$ of either CES or TES-PSA. After $24 \mathrm{~h}$, ethanol was added in large excess in order to destabilise the emulsion. The precipitated solution was then centrifuged and the functionalised nanoparticles were washed twice with ethanol and redispersed in 20 $\mathrm{mL}$ of $\mathrm{RO} / \mathrm{DI}$ water. 


\begin{tabular}{|c|c|c|}
\hline Name & Abbreviation & Molecular structure \\
\hline $\begin{array}{c}\text { 3-(triethoxysilyl)-propylsuccinic } \\
\text { anhydride }\end{array}$ & TES-PSA &
\end{tabular}

158

159

160

161

162

163

164

165

166

167

168

169

170

171

172

173

174

175

176

177

178

179

180

181

182

Table 1: Description of the two silane coupling agents used throughout the study.

\subsection{Characterisation of the nanoparticles}

Hydrodynamic diameters and zeta potentials of the synthesized $\mathrm{SiO}_{2}$ nanoparticles were respectively measured by dynamic light scattering and laser Doppler micro-electrophoresis using a Zetasizer Nano ZS (Malvern Instrument Ltd). The procedure described in Moret et al. was followed (i.e. hydrodynamic diameter was measured five times and zeta potential was measure three time for each samples)[34].

An Hitachi F-2500 fluorescence spectrophotometerwas used to measure photoluminescence properties of the nanoparticles in solution. All measurements were performed at room temperature on samples obtained after redispersing the nanoparticles in water.

\section{$\underline{2.3 \text { Fingermark sampling }}$}

In order to obtain realistic results, and as recommended in the guidelines published by the International Fingerprint Research Group [36], only natural marks were collected. These marks are said to be natural since no enrichment was made on purpose. The only restriction was that the donors did not wash their hands half an hour prior deposition, but were asked to behave normally otherwise. Three donors (one female, two males) were asked to deposit series of marks on four different non-porous substrates (aluminium foils, black polyethylene (PP), transparent polypropylene (PE) and glass). For the optimisation step, single appositions were collected from one donor on aluminium foils. For the comparison step, sets of 20 successive depletive marks were collected for each donor and substrates. The samples were left to age for one week, in an office drawer without specifically controlling the storage conditions. To further test the technique, older marks on aluminium foils, transparent PP and PE were processes as well (aged respectively of 18 months, 2 and 7 years). These marks 
183 came from previous researches for which donors' identity,deposition andstorage conditions were unknown.

\section{$\underline{2.4 \text { Fingermark detection }}$}

187 Various parameters were successively assessed during this study. Three detection 188 conditionswere evaluated and compared, as well as two functional groups containing 189 carboxyl functions and three luminescent dyes (Table 2).

190 Regardless of the chosen parameters, the overall detection protocol consists in a two-step 191 immersion procedure. The items are first immersed in a bath containing the nanoparticles 192 solution for $60 \mathrm{~min}$ (first two detection condition) or $30 \mathrm{~min}$ (third detection condition), then rinsedwith water. The rinsing step is necessary to remove any unwanted nanoparticles remaining on the substrate, which could lead to background noise. Samples are then left to 195 air-dry.

\subsection{Results comparison and evaluation}

203 In order to determine if research on $\mathrm{SiO}_{2}$ nanoparticles application was worth pursuing, the 204 results were compared to a commonly used technique: a one-step luminescent cyanoacrylate 205 the Lumicyano ${ }^{\mathrm{TM}}$ [37]. Each fingermark was cut in half, one half being processed with the 206 nanoparticles solution and the other half fumed with Lumicyano $^{\mathrm{TM}}$ (following the manufacturer's instructions). For each fuming cycle, $1 \mathrm{~g}$ of Lumicyano ${ }^{\mathrm{TM}}$ was placed in MVC1000 fuming cabinet (Foster \& Freeman), at 80\% of relative humidity. The samples were exposed to cyanoacrylate fumes until the detection was considered as optimal (about 15 minutes). Since cyanoacrylate fuming results are generally dependent of the substrate type,

211 each surfacewas processed in a separate cycle.

212 Corresponding halves were then paired again before being photographed in luminescence 213 mode (Note: the luminescence of Lumicyano ${ }^{\mathrm{TM}}$ decreasing with time, the pictures were taken 
214 the day of the fuming process). The imaging conditions were identical for Lumicyano ${ }^{\mathrm{TM}}$ and $215 \mathrm{SiO}_{2}$ nanoparticles, with an excitation at $495 \mathrm{~nm}$ (Minicrimescope MCS400) and an 216 observation at $590 \mathrm{~nm}$ (interferential filter with a band pass of $37 \mathrm{~nm}$ ).

217 Results were assessedfollowing the procedure described by Moret and Bécue [5]. Three 218 independent evaluators were presented one half mark at a time, in a random order. Each half 219 mark was thus assessed independently from its corresponding half. The average scores 220 obtained from each technique and substrate were then compared.

\section{Results and discussion}

\section{$223 \quad \underline{3.1 \mathrm{SiO}_{2}} \underline{\text { nanoparticles synthesis and characterisation }}$}

224 Nanoparticles solutions obtained via micro-emulsion were stable for months when stored at 4 $225{ }^{\circ} \mathrm{C}$ in a fridge.DLS measurements gave an average hydrodynamic diameter of $84.2 \mathrm{~nm}$ and a 226 zeta potential of $-36.7 \mathrm{mV}$ at $\mathrm{pH}$ 6. These values were consistent with those obtained 227 previously[34].

228 Inclusion of dye molecules within the nanoparticles matrix helped obtaining various optical 229 properties (Figure 1). Rhodamine 6G, rhodamine B and RuBpy were selected becausethey 230 covered three distinct areas of the spectrum. They also have been used in previous studies[30$23132]$.

232

233

234

235

236

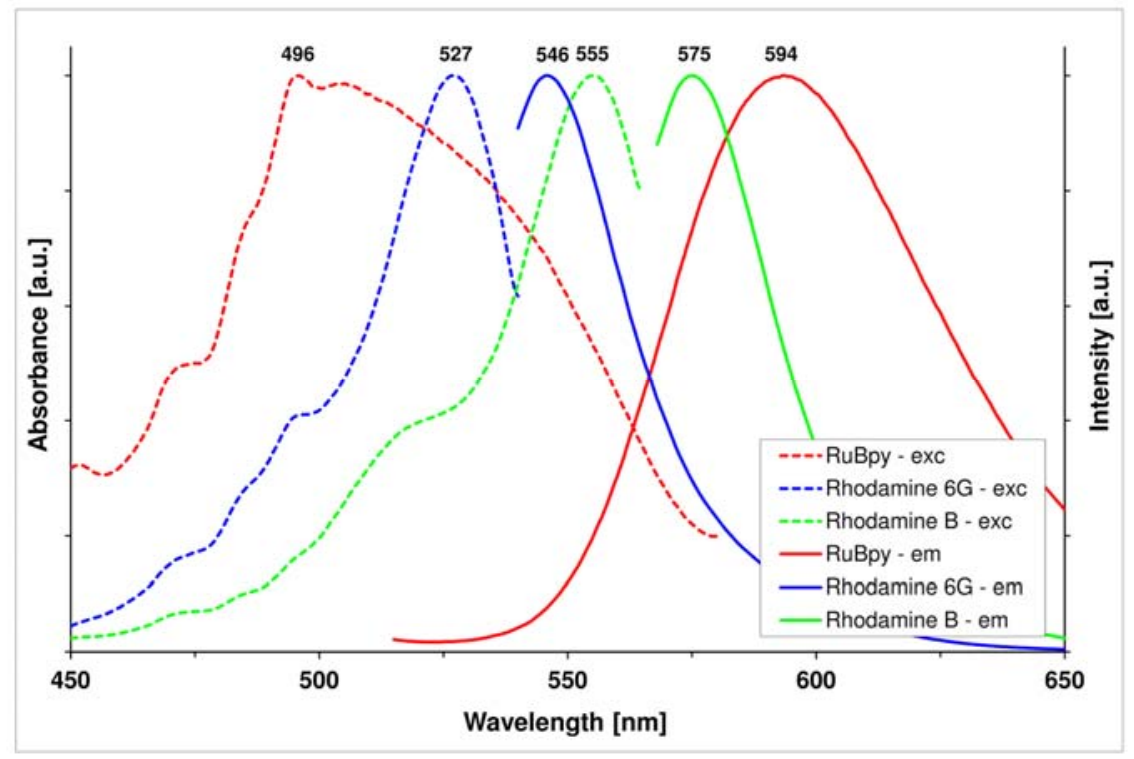

Figure 1: Excitation and emission spectra of silicon oxide nanoparticles solutions synthesised with three different luminescent dyes (RuBpy, rhodamine $6 \mathrm{G}$ and rhodamine $\mathrm{B}$ )

\subsection{Detection mode}


237 Several parameters were compared to obtain better detection results. Three application modes 238 were previously determined to detect mark with success: adjusting the $\mathrm{pH}$ of the 239 nanoparticles solution to 3 , adding sodium chloride $(\mathrm{NaCl})$ to lower the zeta potential 240 intensity and adding a diimide compound to activate the carboxyl groups and favour the 241 interaction with the amine groups of the secretions[34].

242 These three detection modes were compared using the same nanoparticles batch containing 243 rhodamine 6Gand functionalised with CESon fingermarks deposited on aluminium foils 244 (Figure 2). Luminescent results were successfully obtained ineach case.It appeared however 245 that the best results in terms of ridge clarity and luminescence intensity were obtained when $246 \mathrm{NaCl}$ was added in the solution to a concentration of $0.5 \mathrm{M}$. $\mathrm{pH}$ reduction and diimide 247 addition (EDC/NHS) led to slightly inferior results. Addition of $\mathrm{NaCl}$ was consequently 248 chosen as the best detection protocol and considered for the rest of the study.

250

251

252

253

254

255

256

257

258

259

260

261

262
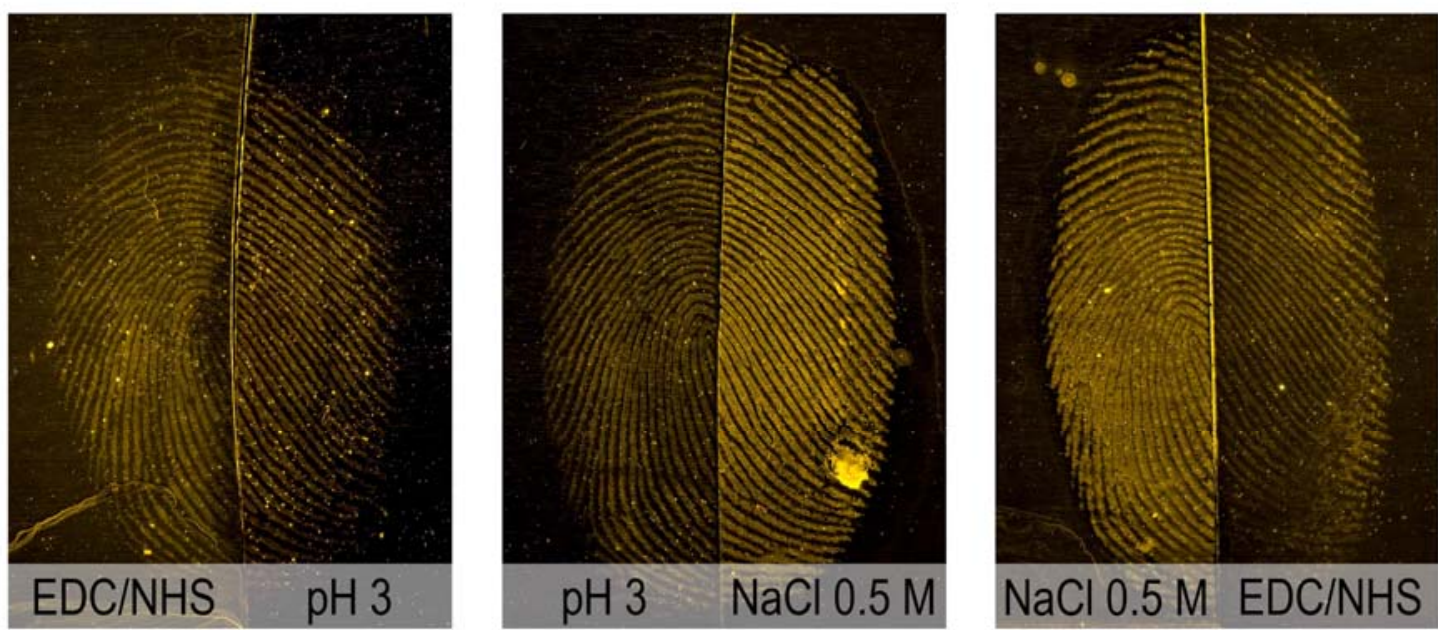

Figure 2: Comparison between three application modes of Rhodamine 6G-dopedSiO $\mathrm{S}_{2}$ nanoparticles solution (adding EDC/NHS, lowering the $\mathrm{pH}$ to 3 and adding $\mathrm{NaCl}$ to a concentration of $0.5 \mathrm{M}$ ). Marks are one-week old, deposited on aluminium foil and visualised in luminescent mode (excitation at $495 \mathrm{~nm}$ and emission at $590 \mathrm{~nm}$ ).

\section{$\underline{3.3 \text { Surface functionalisation }}$}

The second optimisation consisted in selecting the most appropriate function containing carboxyl group that can interact with fingermark residue. Two alkoxysilanesweregrafted on the surface of nanoparticles: CES and TES-PSA (Table 1). They both contain carboxyl group, but the second one doubles the amount of functional groupspresent on the nanoparticle surface, due to the opening of the succinic chain in water. Since carboxyl groups drive the nanoparticles interaction with secretions, TES-PSAcould lead to a better detection due to the 
263 larger amount of reactive groups. Comparisons were made between two solutions containing nanoparticles functionalised respectively withCES and TES-PSA. As determined previously, $\mathrm{NaCl}(0.5 \mathrm{M})$ was added to favour the interaction. A significantly higher background noise appeared with TES-PSAfunctionalised nanoparticles and the obtained marks appeared blurred(Figure 3). With CES, an obvious quality difference was observed.Sharp luminescent ridges were obtained, with clear level 3 features. Since CES functionalised nanoparticles gave the best results, they were selected for this study.

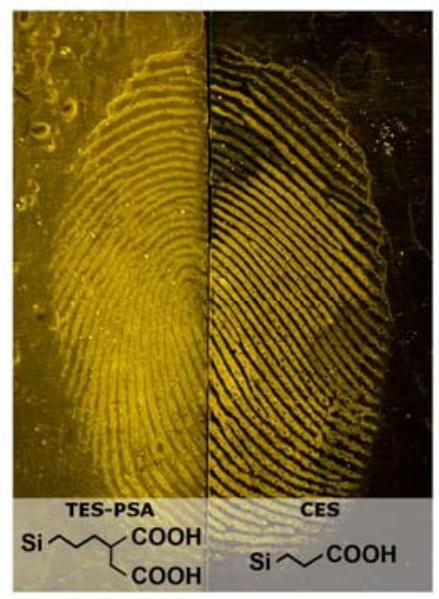

Figure 3: Comparison between Rhodamine 6G-doped silicon oxide nanoparticles functionalised withTES-PSA((triethoxysilyl)-propylsuccinic anhydride - left half) and CES(carboxyethylsilanetriol - right half), and used to detect fingermarks. The illustrated fingermark is one-week old, deposited on aluminium foil and visualised in luminescent mode (excitation at $495 \mathrm{~nm}$ and emission at $590 \mathrm{~nm}$ ).

\subsection{Luminescence properties}

The last optimisation step was about the nanoparticles' optical properties. As described above, three dyes were considered: rhodamine $6 \mathrm{G}$ used as a reference, rhodamine $\mathrm{B}$ and RuBpy. Each half mark was observed under the luminescent conditions that lead to the best result. RuBpy was illuminated at $495 \mathrm{~nm}$, whereas both rhodamine $6 \mathrm{G}$ and rhodamine $\mathrm{B}$ were excited at $515 \mathrm{~nm}$. All marks were observed at $590 \mathrm{~nm}$. These conditions differed from the optimal conditions determined by spectrofluorimetry. If the theoretical conditions were to be respected, the small Stokes shift of rhodamine $6 \mathrm{G}$ and B would lead to a background noise due to the excitation wavelength. Based on the spectrofluorometry results (Figure 1), RuBpy appeared to be the best choice since it has the larger Stokes shift $(100 \mathrm{~nm})$ compared to the other two dyes $(\sim 20 \mathrm{~nm})$. These conditions were the one resulting in the best luminescence intensity with the lowest background.Rhodamine $6 \mathrm{G}$ and rhodamine $\mathrm{B}$ showed equivalent 
290 results in terms of luminescence intensity (Figure 4). RuBpypresented a much more intense

291 luminescence, compared to the two other dyes. It led to a light background staining, but since

292 RuBpyhad a larger Stokes shift and the intensity of the final results was stronger, it was

293 chosen as the most suitable dye for this study. The observed background noise obtained after

294 immersing the samples was not judged detrimental to fingermark visualisation.

296

297

298

299

300

301

302

303

304

305

306

307

308

309

310

311 Conventional cyanoacrylate fuming is one of the most commonly used techniques for the

312 detection of fingermarks on non-porous substrates. However, it cannot be directly compared

313 to $\mathrm{SiO}_{2}$ nanoparticles sincethe obtained results are not luminescent.In order to avoid

314 overestimating results and introducing a bias toward one technique or the other, a
Figure 4: Comparison between $\mathrm{SiO}_{2}$ nanoparticles synthetised with three different luminescent dyes (rhodamine 6G, rhodamine B and RuBpy). Marks are one-week old, deposited on aluminium foil and visualised in luminescent mode (excitation at $495 \mathrm{~nm}$ and emission at $590 \mathrm{~nm}$ ).
These optimisation experimentsled to select the parameters leading to the best detection quality. Optimal detection was thus obtained with silicon oxide nanoparticles containing RuBpy, functionalised with CES and applied in a solution at $\mathrm{pH} 6$ containing $0.5 \mathrm{M}$ of sodium chloride for $60 \mathrm{~min}$.Should this technique be applied on a larger scale, further improvement would be needed, in order to reduce the immersion timefor example. However, results obtained so far constitute a valid base to conduct a comparison with a benchmark technique.

\subsection{Comparison with cyanoacrylate fuming}


315 luminescent technique should therefore be used to offer a valid comparison.Luminescent 316 marks can be obtained using conventional cyanoacrylate by following a two-step procedure 317 consisting in fumigating the samples first, then dye-stainingthem with a luminescent dye 318 dissolved in a solvent[38].However, to avoid tedious staining procedures and to obtain more 319 homogeneous results throughout the sample sets, a one-step luminescent cyanoacrylate was 320 privileged during this study: theLumicyano ${ }^{\mathrm{TM}}[37]$. The obtained marks offer luminescent 321 properties either under UV $(326 \mathrm{~nm})$ or at $511 \mathrm{~nm}$ and the results can be observed with an 322 emission band centred at $562 \mathrm{~nm}$. It however appeared that good luminescent marks could be 323 observed under the same conditions used for RuBpy (i.e. excitation at $495 \mathrm{~nm}$ and emission 324 filter at $590 \mathrm{~nm}$ ). Using the same visualisation conditions help to image both sides at the 325 same time and to keep constant parameters for comparison.

326 Both techniques were applied on sets of 20 depletive marks from 3 donors on 4 different 327 substrates (a total of 240 marks) as described in the materials and methods section. The 328 samples were processed and imaged the same day in order to avoid any age inconsistency, or 329 luminescence intensity loss. Both techniques have been applied according to the established 330 procedure and led to satisfactory results. Under white light, the results obtained with 331 Lumicyano ${ }^{\mathrm{TM}}$ were similar to those that can be obtained with common cyanoacrylate.

332 Before discussing the sensibility obtained on depletive marks, the results obtained on the first 333 finger apposition are presented(Figure 5). Since results quality is related to the nature of the 334 substrate, they will be described separately. Aluminium foil is generally considered as an 335 idealsurfaceallowing good results to be obtained. Luminescent results were obtained for both 336 techniques, with a stronger intensity for Lumicyano ${ }^{\mathrm{TM}}$ (Figure 5 - first column). However, 337 when ridge detailswere considered, it can be seen that cyanoacrylate-fumed marks tended to 338 give dotted marks with ridge-continuity disruption. This was especially the case for donors A 339 and $\mathrm{B}$, but not for donor $\mathrm{C}$ for which very homogenous results were obtained.This 340 phenomenon was however not observed for the fingermarks detected by $\mathrm{SiO}_{2}$ nanoparticles.

341 Even if marks from donor B were not fully detected, nanoparticles developed marks with a 342 higher homogeneity for the three donors. An affinity of nanoparticles for the aluminium foil 343 was observed, leading to a slight background staining. This phenomenon, already noticed 344 during the optimisation phase, did not prevent fingermarks observation. The background was 345 not due to a lack of rinsing since it appears uniformly on the entire surface. It is most likely 346 due to unwanted interactions with the substrate. This issue could potentially be reduced by a 347 shorter immersion time in the nanoparticles solutions, and should be further studied. By 348 comparison, no background apparition was noticed with cyanoacrylate fuming. 
349 Regarding black PE, the previous observations were still valid(Figure 5 - second column).

350 Cyanoacrylate luminescence was less intense and led again to doted marks. For donor B, the 351 markswere barely visible. The background noise observed with $\mathrm{SiO}_{2}$ on aluminium foil is 352 much more pronounced on PE.Sometimes it concealed the marks almost entirely (donors A 353 and B).It was however not homogeneous and when absent it became possible to observe 354 luminescent marks of high quality with clear level 3 features (donor C). Nanoparticles were 355 thus effectively attracted on the secretions, but the ridges were partially covered by a non356 selective deposition onto the surface. That reinforces the needs of further studies on the 357 interaction with substrates.

358 Transparent PP provided significantly different results compared to the two previous 359 substrates(Figure 5 - third column). For the cyanoacrylate, luminescent marks were obtained 360 for both donor $\mathrm{A}$ and $\mathrm{C}$, but the detection was not homogeneous towards the edge of the 361 donor A's mark and almost no ridges were visible for the donor B. The situation was quite 362 different for the $\mathrm{SiO}_{2}$ nanoparticles; the detection itself was very homogeneous for all three 363 donors in terms of mark quality, with no background staining observed. .

364 On glass, the nanoparticles solution haslargely washed away the marks(Figure 5 365 secondlast). Only a weak luminescence and low quality markswere observed. On the other 366 halves, cyanoacrylate performed well for the three donors, even if a light background staining 367 appeared. Considering $\mathrm{SiO}_{2}$ nanoparticles, the detection protocol seems not appropriate for 368 this type of substrate and should thus be further adjusted. Reduction of immersion time or 369 finding another carrier solvent could be key factors that could lead to better results.

370 In summary, for the first marks of the depletion series, cyanoacrylate succeeded in detecting 371 marks on all tested surfaces. However, differences in quality were consistently observed 372 between donors. These variations can be attributed to the quantity and quality variation 373 among donors' secretions. However, this trend was less pronounced with $\mathrm{SiO}_{2}$ nanoparticles 374 that react not with specific compounds of the secretion, but with specific functional groups. 375 The results quality washowever more tightly related to the substrate type, which indicates that 376 the detection conditions can be further adjusted. Glass appeared to be a challengingsurface 377 since nanoparticles solution tended to wash the secretion, and thus failing in detecting any 378 mark. Moreover, background staining appeared on black PE, impinging the detection ofthe 379 marks. Future optimisations need to be carried on, especially to decrease the unwanted 380 background staining and to obtain a more versatile technique that could be applied on a wider 381 range of substrates. $\mathrm{SiO}_{2}$ nanoparticles application remains promising since the obtained 
382 results are very homogeneous and appeared to be less donor-dependent. This, in itself, 383 represents a considerable advantage over cyanoacrylate fuming.

384
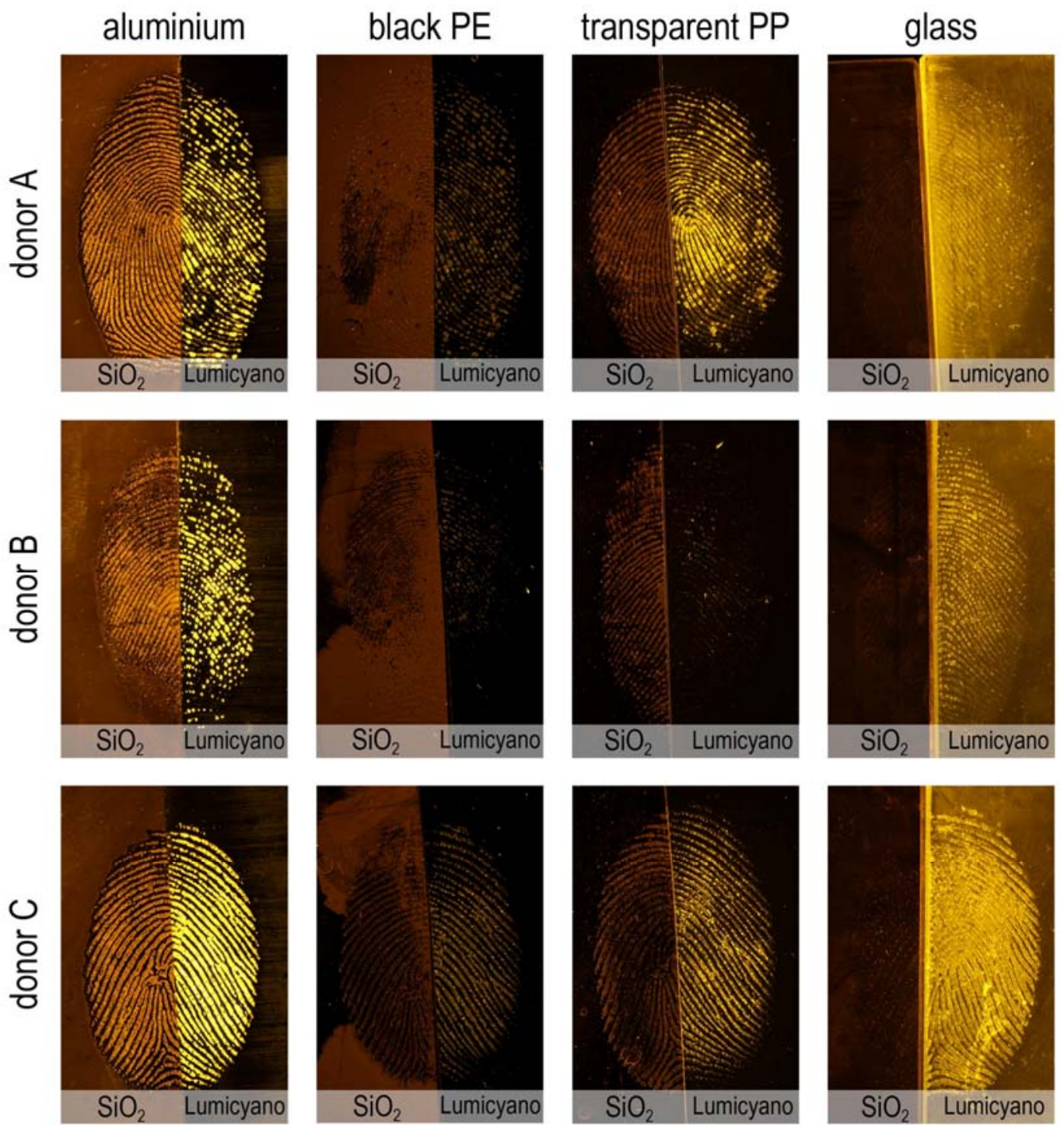

Figure 5: Comparison of fingermarks detected with RuBpy-doped silicon oxide nanoparticles

389 Results obtained with the depletive series are presented below. On average, by taking into

390 account every mark from each donor and substrate, comparable scores were obtained for both

391 techniques (1.3 for Lumicyano ${ }^{\mathrm{TM}}$ and 1.5 for $\mathrm{SiO}_{2}$ nanoparticles) (Figure 6). When only the

392 first five depletions were considered, a general increase of scoreswas noticed (1.8 for

393 Lumicyano $^{\mathrm{TM}}$ and 2.1 for $\mathrm{SiO}_{2}$ nanoparticles), showing that nanoparticles gave slightly better

394 resultson average (Figure 6). 
400 By looking at each substrate separately and considering only the first five depletions, results 401 can be described in more details (Figure 7). On aluminium foil, equivalent results were 402 403 404 405 406 407 408 409

Figure 6: Comparison of the average results obtained with Lumicyano ${ }^{\mathrm{TM}}(\mathrm{CA})$ and RuBpydoped $\mathrm{SiO}_{2}$ nanoparticles. obtained showing that both techniques performed well, but on glass, as described above, $\mathrm{SiO}_{2}$ solution tends to wash the marks, leading to low quality results, cyanoacrylate fuming showssuperior scores. For the black PE, even if background staining was observed, the average quality was still superior for $\mathrm{SiO}_{2}$ nanoparticles compared to cyanoacrylate. The biggest difference appeared on transparent PP where cyanoacrylate stopped being effective after just a few depletions, while $\mathrm{SiO}_{2}$ nanoparticles kept detecting marks. This was not only true for the first five depletions, good quality results were obtained until the twentieth depletion (Figure 8).

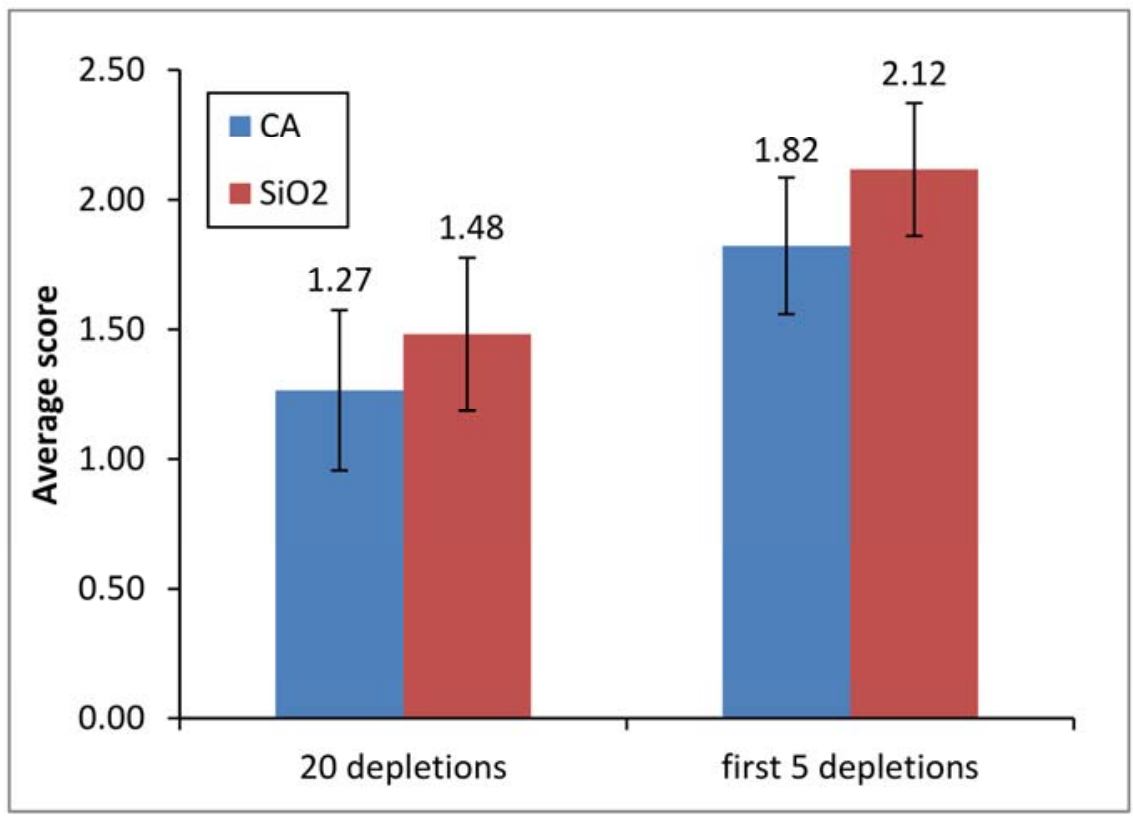




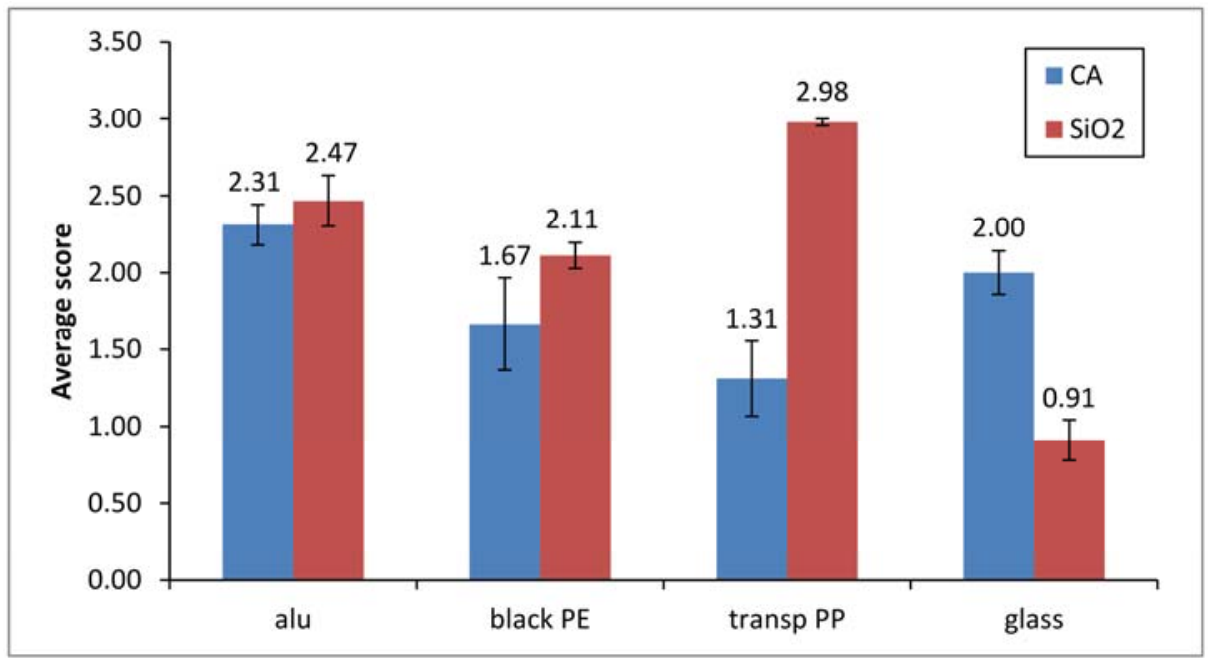

Figure 7: Comparison of the average results obtained with Lumicyano ${ }^{\mathrm{TM}}(\mathrm{CA})$ and RuBpydoped $\mathrm{SiO}_{2}$ nanoparticles on four different substrates.
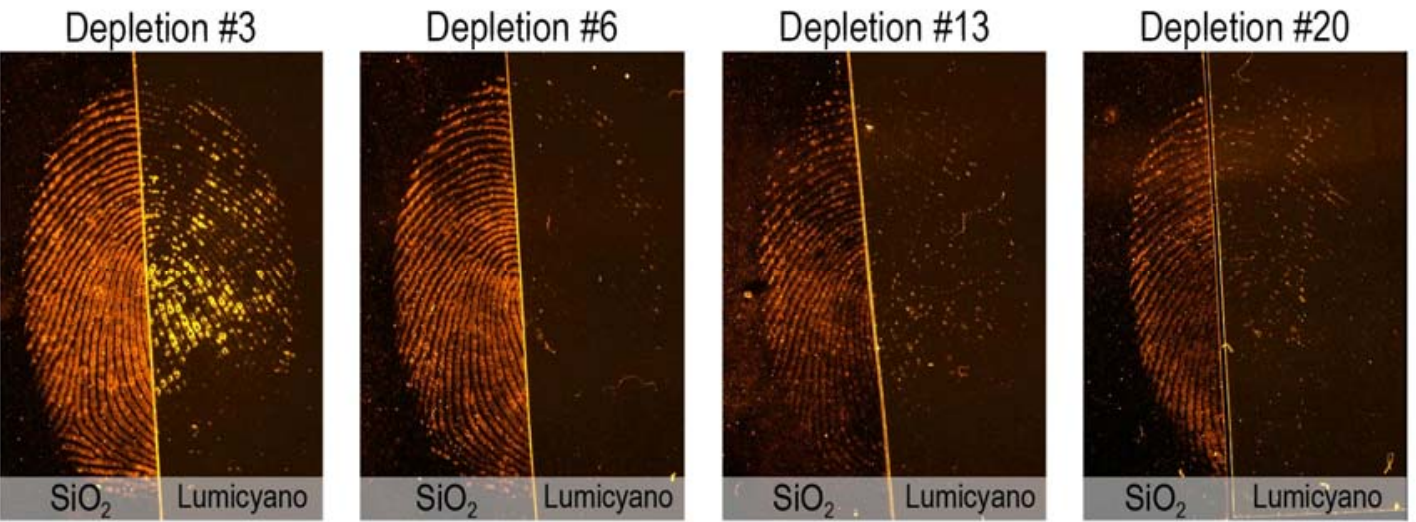

Figure 8:Comparison of depletive fingermarks detected with RuBpdy-doped silicon oxide nanoparticles (left halves) and luminescent cyanoacrylate (right halves) on transparent PP.

When average scores were calculated considering each donor individually, $\mathrm{SiO}_{2}$ nanoparticles were less donor dependent that cyanoacrylate (Figure 9). The calculated variance between donors for cyanoacrylate is 0.41 , whereas the one for $\mathrm{SiO}_{2}$ nanoparticles is only 0.02 .

To compare the efficiency of the two techniques over the entire depletion series, marks of a score above or equal to 2 were added (Figure 10). Cyanoacrylate on aluminium detected the greatest amount of marks. $\mathrm{SiO}_{2}$ nanoparticles solution, as detailed above, was not efficient on glass, but detected a comparable amount of marks on the three other substrates. 


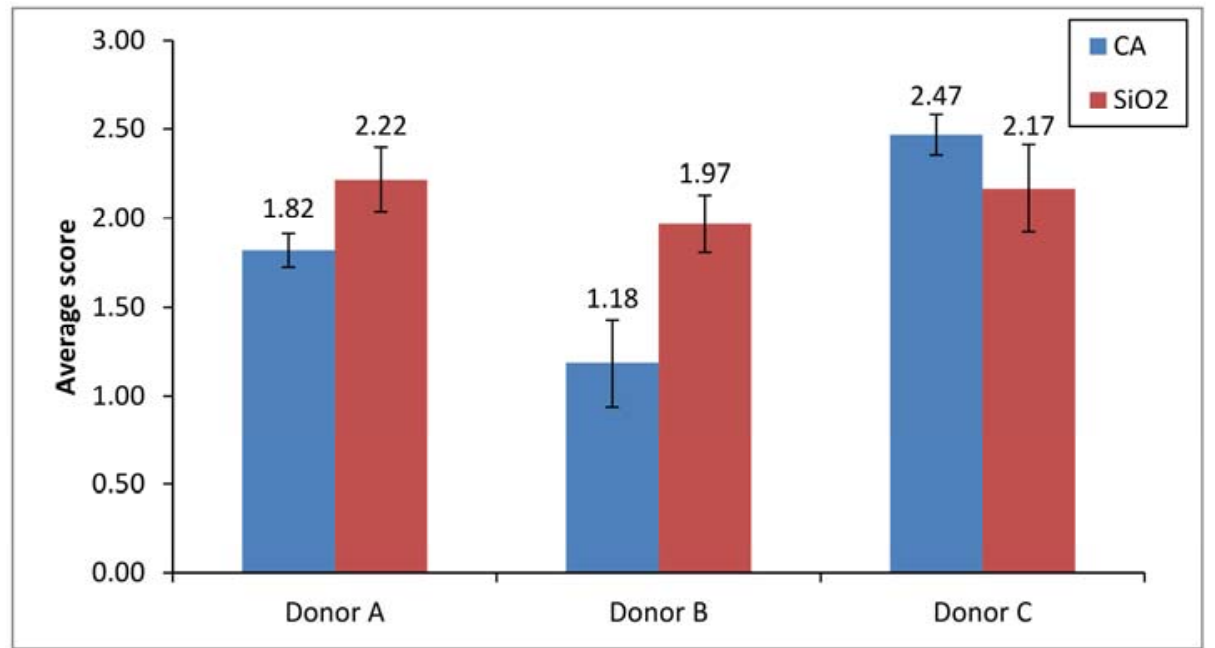

Figure 9: Comparison of the average results obtained from three different donors withLumicyano ${ }^{\mathrm{TM}}(\mathrm{CA})$ and $\mathrm{RuBpy}$-doped $\mathrm{SiO}_{2}$ nanoparticles.

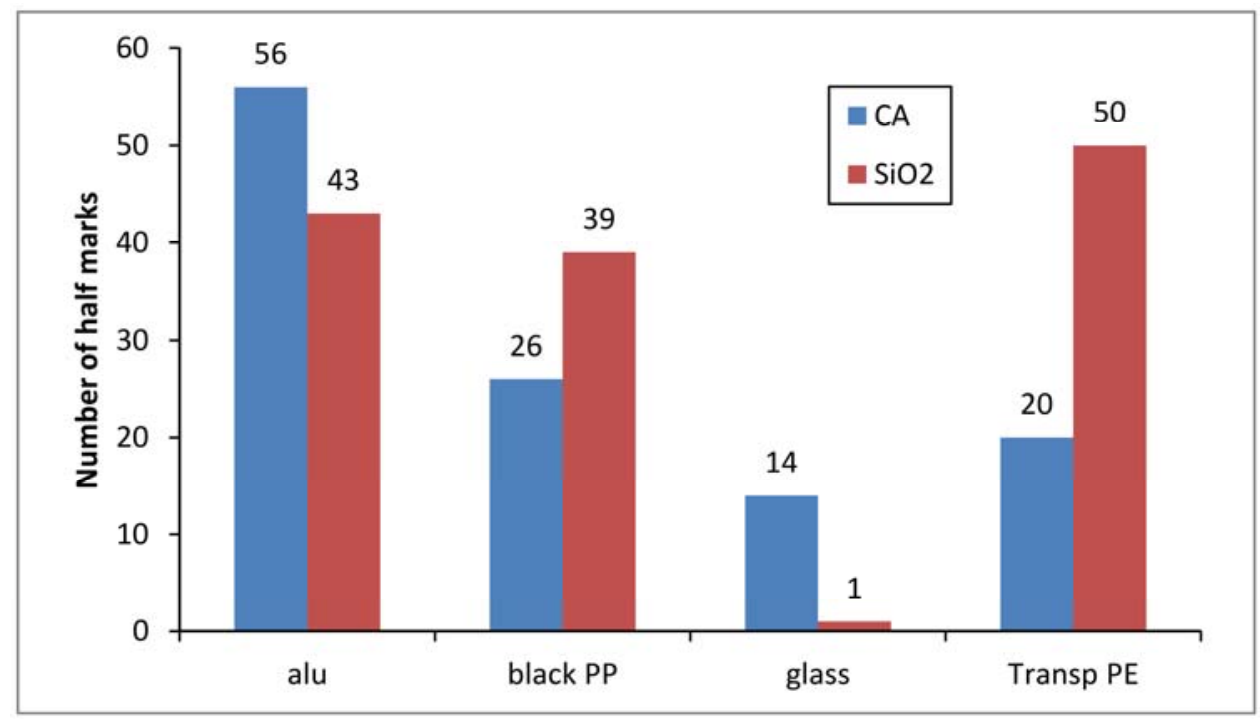

Figure 10:Comparison between the amount of mark with a score equal or above 2 detected per substrate for each technique.

To conclude the comparisons, aged marks on aluminium foils, transparent PP and PE were process following the same established protocol. Both cyanoacrylate fuming and $\mathrm{SiO}_{2}$ nanoparticles solution were able to detect marks as old as 7 years. On aluminium foils, $\mathrm{SiO}_{2}$ nanoparticles tended to give better ridge details. The reversed situation was observed on transparent PP. For the 7 years marks, Lumicyano ${ }^{\mathrm{TM}}$ was superior on transparent PP, with much better ridge quality. On $\mathrm{PE}$, more completed marks were detected with $\mathrm{SiO}_{2}$ nanoparticles, despite missing information in the centre, whereas cyanoacrylate presented good details on the upper part and very few details on the lower part. 

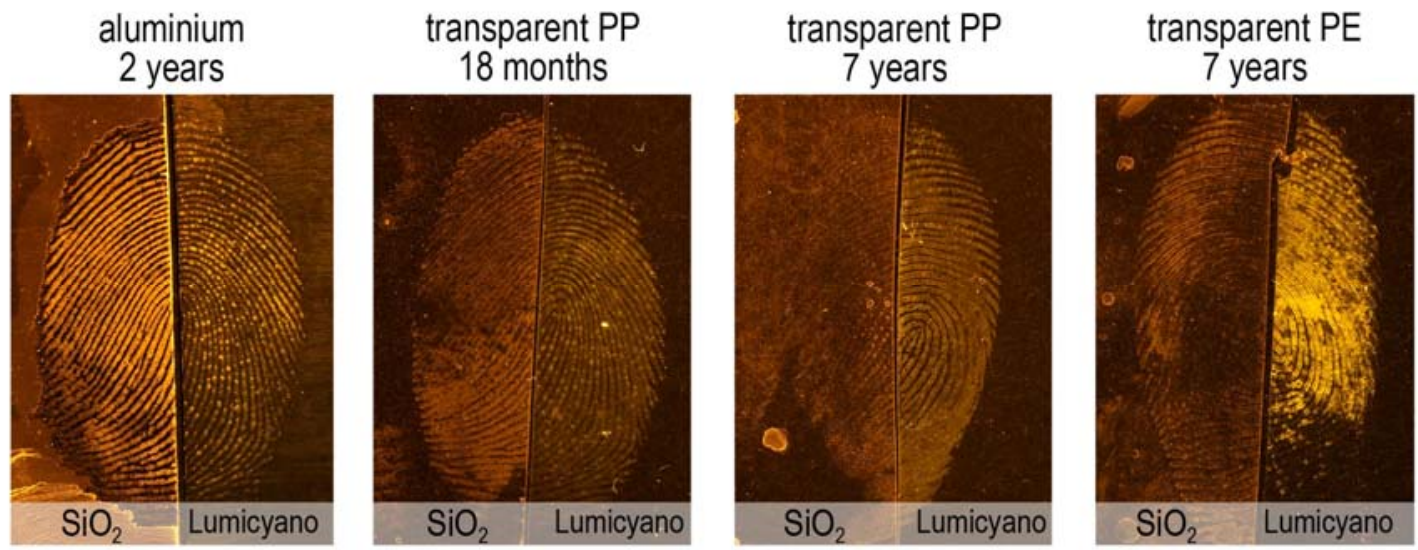

Figure 11:Comparison of old fingermarks (18 months to 7 years) detected with RuBpy-doped

\section{Conclusions}

450 This paperinvestigated an application of luminescent functionalised $\mathrm{SiO}_{2}$ nanoparticles for

451 fingermark detection. Several optimisations of the detection protocol for considering dye452 doped $\mathrm{SiO}_{2}$ nanoparticles as efficient fingermark reagents were presented. Appropriate 453 detection conditions, outer functionalisations and luminescent dyes were successively studied 454 and selected, leading to an optimised protocol. A comparison to Lumicyano ${ }^{\mathrm{TM}}$, a one-step 455 luminescent cyanoacrylate (CA), was then conducted to assess the efficiency of the method. 456 Both techniques were compared on series of 20 depletive marks from three donors on four 457 different non-porous substrates.

458 On average, both techniques performed similarly.However, for Lumicyano ${ }^{\mathrm{TM}}$, quality 459 variations between donors were observed. Interestingly, that was not observed (or only to a 460 limited extend) for $\mathrm{SiO}_{2}$ nanoparticles. This might be due to the fact that the technique targets 461 a specific functional group instead of particular compounds of the secretion. $\mathrm{The}^{\mathrm{SiO}_{2}}$ results 462 quality was instead more related to the substrate type.

463 Heterogeneous detection results proved that the technique remained to be further optimised.

464 Regarding health and safety issue aboutSiO${ }_{2}$ nanoparticles application, no toxicity assessment 465 of the synthesized nanoparticles was performed in this study. This should be considered in 466 details before any large scale application.More work still needs to be undertaken in order to 467 provide a fully operational technique, but the reported results in this study showed that $\mathrm{SiO}_{2}$ 468 nanoparticles are very promising and that research effort should be further pursued. 


\section{References}

472 [1] Bécue A., Moret S., Champod C., Margot P. (2011), Use of stains to detect

[2] Chadwick S., Maynard P., Kirkbride P., Lennard C., Spindler X., Roux C. (2011), Use of styryl 11 and STaR 11 for the luminescence enhancement of cyanoacrylatedeveloped fingermarks in the visible and near-infrared regions, Journal of Forensic Sciences, 56, 1505-1513.

[3] Leggett R., Lee-Smith E. E., Jickells S. M., Russell D. A. (2007), "Intelligent" fingerprinting: Simultaneous identification of drug metabolites and individuals by using antibody-functionalized nanoparticles, Angewandte Chemie International Edition, 46, 4100-4103.

[4] Schnetz B., Margot P. (2001), Technical note: Latent fingermarks, colloidal gold and multimetal deposition (MMD) optimisation of the method, Forensic Science International, 118, 21-28.

[5] Moret S., Bécue A. (2015), Single-metal deposition for fingermark detection-a simpler and more efficient protocol, Journal of Forensic Identification, 65, 118-137.

[6] Cantú A. A., Johnson J. L. (2001), Silver physical development of latent prints, In: Advances in fingerprint technology, 2nd ed., Lee, H. C., Gaensslen, R. E., Eds., CRC Press: Boca Raton, FL; pp 241-274.

[7] Wade D. C. (2002), Development of latent prints with titanium dioxide $\left(\mathrm{TiO}_{2}\right)$, Journal of Forensic Identification, 52, 551-559.

[8] Sodhi G. S., Kaur J. (2006), Nanoparticle size fingerprint dusting composition based on fluorescent eosin Y dye, Fingerprint Whorld, 32, 146-147.

[9] Sodhi G. S., Kaur J. (2007), A novel, nanoparticle size fingerprint detecting composition based on fluorescent lucifer Y stain, Fingerprint Whorld, 34, 24-25.

[10] Choi M. J., McBean K. E., Ng P. H. R., McDonagh A. M., Maynard P. J., Lennard C., Roux C. (2008), An evaluation of nanostructured zinc oxide as a fluorescent powder for fingerprint detection, Journal of Materials Science, 43, 732-737.

[11] Bécue A., Moret S., Champod C., Margot P. (2009), Use of quantum dots in aqueous solution to detect blood fingermarks on non-porous surfaces, Forensic Science International, 191, 36-41.

[12] Dilag J., Kobus H., Ellis A. V. (2009), Cadmium sulfide quantum dot/chitosan nanocomposites for latent fingermark detection, Forensic Science International, 187, 97-102.

[13] Dilag J., Kobus H. J., Ellis A. V. (2011), Nanotechnology as a new tool for fingermark detection: A review, Current Nanoscience, 7, 153-159.

[14] Bécue A., Cantú A. A. (2012), Fingermark detection using nanoparticles, In: Lee and Gaensslen's advances in fingerprint technology, 3rd ed., Ramotowski, R. S., Ed., CRC Press LLC; pp 307-379.

[15] ASTM International (2006), Standard terminology relating to nanotechnology; ASTM International, Ed.; Vol. E 2456 - 06.

[16] British Standards Institution (2005), Vocabulary - nanoparticles; British Standards Institution, Ed.; Vol. PAS 71:2005.

[17] Bécue A., Scoundrianos A., Champod C., Margot P. (2008), Fingermark detection based on the in situ growth of luminescent nanoparticles - towards a new generation of multimetal deposition, Forensic Science International, 179, 39-43. 
[18] Chadwick S., Maynard P., Kirkbride P., Lennard C., McDonagh A., Spindler X., Roux

[19] Alivisatos A. P. (1996), Semiconductor clusters, nanocrystals and quantum dots, Science, 271, 933-937.

[20] Bukowski T. J., Simmons J. H. (2002), Quantum dot research: Current state and future prospects, Critical Reviews in Solid State and Materials Sciences, 27, 119-142.

[21] Menzel R. E. (2000), Photoluminescence detection of latent fingerprints with quantum dots for time-resolved imaging, Fingerprint Whorld, 26, 119-123.

[22] Cheng K. H., Ajimo J., Chen W. (2008), Exploration of functionalized CdTe nanoparticles for latent fingerprint detection, Journal of Nanoscience and Nanotechnology, 8, 1170-1173.

[23] Moret S., Bécue A., Champod C. (2013), Cadmium-free quantum dots in aqueous solution: Potential for fingermark detection, synthesis and an application to the detection of fingermarks in blood on non-porous surfaces, Forensic Science International, 224, 101-110.

[24] Yu X., Liu J., Zuo S., Yu Y., Cai K., Yang R. (2013), Application of mercaptosuccinic acid capped CdTe quantum dots for latent fingermark development, Forensic Science International, 231, 125-130.

[25] Wang Y. F., Yang R. Q., Shi Z. X., Liu J. J., Zhao K., Wang Y. J. (2014), The effectiveness of CdSe nanoparticle suspension for developing latent fingermarks, Journal of Saudi Chemical Society 18, 13-18.

[26] Xu C., Zhou R., He W., Wu L., Wu P., Hou X. (2014), Fast imaging of eccrine latent fingerprints with nontoxic mn-doped ZnS QDs, Analytical Chemistry, 86, 3279-3283.

[27] Gao F., Han J., Zhang J., Li Q., Sun X., Zheng J., Bao L., Li X., Liu Z. (2011), The synthesis of newly modified CdTe quantum dots and their application for improvement of latent fingerprint detection, Nanotechnology, 22, art. No. 075705 (10 pp.).

[28] Stöber W., Fink A., Bohn E. (1968), Controlled growth of monodisperse silica spheres in the micron size range, Journal of Colloid and Interface Science, 26, 62-69.

[29] López-Quintela A. (2003), Synthesis of nanomaterials in microemulsions: Formation mechanisms and growth control, Current Opinion in Colloid and Interface Science, 8 , 137-144.

[30] Theaker B. J., Hudson K. E., Rowell F. J. (2008), Doped hydrophobic silica nano- and micro-particles as novel agents for developing latent fingerprints, Forensic Science International, 174, 26-34.

[31] Liu L., Gill S. K., Gao Y., Hope-Weeks L. J., Cheng K. H. (2008), Exploration of the use of novel $\mathrm{SiO}_{2}$ nanocomposites doped with fluorescent $\mathrm{Eu}^{3+} /$ sensitizer complex for latent fingerprint detection, Forensic Science International, 176, 163-172.

[32] Liu L. (2011), Study on the use of rhodamine doped nanocomposite for latent fingerprint detection, Advanced Materials Research, 295-297, 813-816.

[33] Huang W., Li X., Wang H., Xu X., Liu H., Wang G. (in press), Synthesis of amphiphilic silica nanoparticles for latent fingerprint detection, Analytical Letters.

[34] Moret S., Bécue A., Champod C. (2014), Nanoparticles for fingermark detection: An insight into the reaction mechanism, Nanotechnology, 25, 425502.

[35] Bagwe R. P., Yang C., Hilliard L. R., Tan W. (2004), Optimization of dye-doped silica nanoparticles prepared using a reverse microemulsion method, Langmuir, 20, 83368342.

[36] International Fingerprint Research Group (IFRG) (2014), Guidelines for the assessment of fingermark detection techniques, Journal of Forensic Identification, 64, 174-200. 
566

567

568

569

570

[37] Prete C., Galmiche L., Quenum-Possy-Berry F.-G., Allain C., Thiburce N., Colard T. (2013), Lumicyano ${ }^{\mathrm{TM}}$ : A new fluorescent cyanoacrylate for a one-step luminescent latent fingermark development, Forensic Science International, 233, 104-112.

[38] Mazzella W. D., Lennard C. (1995), An additional study of cyanoacrylate stains, Journal of Forensic Identification, 45, 5-18.

571 